Planetary Systems in the Universe - Observation, Formation and Evolution

Proceedings IAU Symposium No. 202, (C)2004 IAU

Alan Penny, Pawel Artymowicz, Anne-Marie Lagrange, 6 Sara Russell, eds.

\title{
Minimum Mass of Brown Dwarfs
}

\author{
Taishi Nakamoto \\ University of Tsukuba, Japan
}

Koji Ogochi

Information Technology Japan Inc., Japan

\begin{abstract}
We investigate the minimum mass of brown dwarfs using radiation hydrodynamics simulations of collapsing filamentary clouds and analytical estimates. We found the minimum mass of brown dwarfs depends on the initial temperature of parent clouds and the nature of heating sources. The universal minimum mass of brown dwarfs, which is about $0.007 M_{\odot}$, is given when the initial cloud temperature is $13.3 \mathrm{~K}$. Our results are consistent with recent observations of low-mass objects in some star forming regions.
\end{abstract}

\section{Introduction}

What are planets? How different are they from brown dwarfs? Since the mass of planets and brown dwarfs are likely to be mainly determined by their formation processes, it is worth investigating the minimum mass of brown dwarfs that may be used to distinguish planets from brown dwarfs.

We use a definition of brown dwarfs as objects formed by the self gravitational collapse of interstellar clouds. And we define planets as objects formed through other mechanisms, e.g., accumulation of small solid objects, etc. The bulk composition of brown dwarfs is expected to be equal to that of interstellar clouds, whereas that of planets can be different.

The minimum mass of brown dwarfs has been studied by several authors (Rees 1976; Silk 1977; Inutsuka \& Miyama 1997 (IM97); Masunaga \& Inutsuka 1999). We extend the study into more general one especially regarding the energy transfer processes and the initial/boundary conditions.

\section{Theoretical Scenario and Model of Brown Dwarf Formation}

It is known that a gravitationally unstable cloud without any rotation and magnetic fields is likely to collapse and form a sheet-like cloud. The sheet-like cloud is likely to fragment into filamentary clouds (Miyama, Narita, \& Hayashi 1984). And a filament shrinks thinner and thinner without any fragmentation if it keeps isothermal (IM97). However, when the temperature of the filament starts to rise due to the compressional heating accompanying to the collapse, the collapse in the radial direction stops. Since the filament formed through those processes 
are unstable against motion along the axis of the filament, the filament begins to fragment into many pieces (IM97); they are considered to be brown dwarfs.

In order to evaluate the mass of brown dwarfs, we examine the critical density of filaments at which the filaments stop collapsing in the radial direction, because the fragment mass can be estimated using the density by the linear stability analysis (IM97). We carry out the numerical simulation of filamentary clouds using one dimensional radiation hydrodynamics. In our simulations, the radiative energy transfer is solved very accurately because it influences the cloud temperature, pressure, and eventually the dynamics of the cloud significantly. We ignore the rotation and magnetic fields of the cloud, and the accumulation of fragments; they can increase the mass of fragments. Therefore, we can have the minimum mass of fragments, i.e., brown dwarfs with our analysis.

We assume the filamentary cloud is heated by (a) the stellar radiation or (b) cosmic rays, and maintains constant temperature initially. The opacity of the cloud gas is assumed to be $\kappa=2 \times 10^{-2}(T / 10 \mathrm{~K})^{2} \mathrm{~cm}^{2} \mathrm{~g}^{-1}$ where $T$ is the gas temperature. The cloud can be opaque to the radiation, while the cloud is assumed to keep transparent to the cosmic ray. The heating rates due to (a) the stellar radiation and (b) cosmic rays are represented in terms of the initial gas temperature. Details of our model and computational method are described in Ogochi \& Nakamoto (2000).

\section{Results and Discussions}

Combining results of our numerical simulations of collapsing filamentary clouds and analytical considerations, we obtained the minimum mass of brown dwarfs, which are shown in Figure 1. The minimum mass of brown dwarfs depends on the initial cloud temperature and the heating source. In other words, it depends on the environment of parent clouds. The universal minimum mass, which is about $0.007 M_{\odot}$, is given when the initial cloud temperature is $13.3 \mathrm{~K}$.

When clouds with $T_{\text {init }}<T_{s}$ stop radial collapses, they are still transparent to the radiation, while clouds with $T_{\text {init }}>T_{s}$ are opaque when they do. It should be pointed out that if the cloud is heated by the stellar radiation, the time when the cloud stops radial collapse is NOT equal to the time when the cloud becomes opaque. If the cloud is heated by cosmic rays and $T_{\text {init }}>T_{s}$, the cloud radial collapse stops when the cloud becomes opaque.

According to recent observations, it is found that the minimum mass of lowmass objects in some star forming regions is about $0.01 M_{\odot}$ (Zapatero Osorio et al. 1999; Luhman et al. 2000; Hillenbrand \& Carpenter 2000). This fact is quite consistent with our results when we remember that the typical cloud temperature of star forming regions is about $10 \mathrm{~K}$. Moreover, it is reported that the minimum mass of objects in the inner Orion Nebula Cluster is likely to be higher than $0.01 M_{\odot}$ (Hillenbrand \& Carpenter 2000). This seems also natural because the cloud temperature in that region is expected to be higher than 10 $\mathrm{K}$ and that leads the higher fragment mass according to our results.

\section{References}

Hillenbrand, L. A., \& Carpenter, J. M. 2000, ApJ, 540, 236 


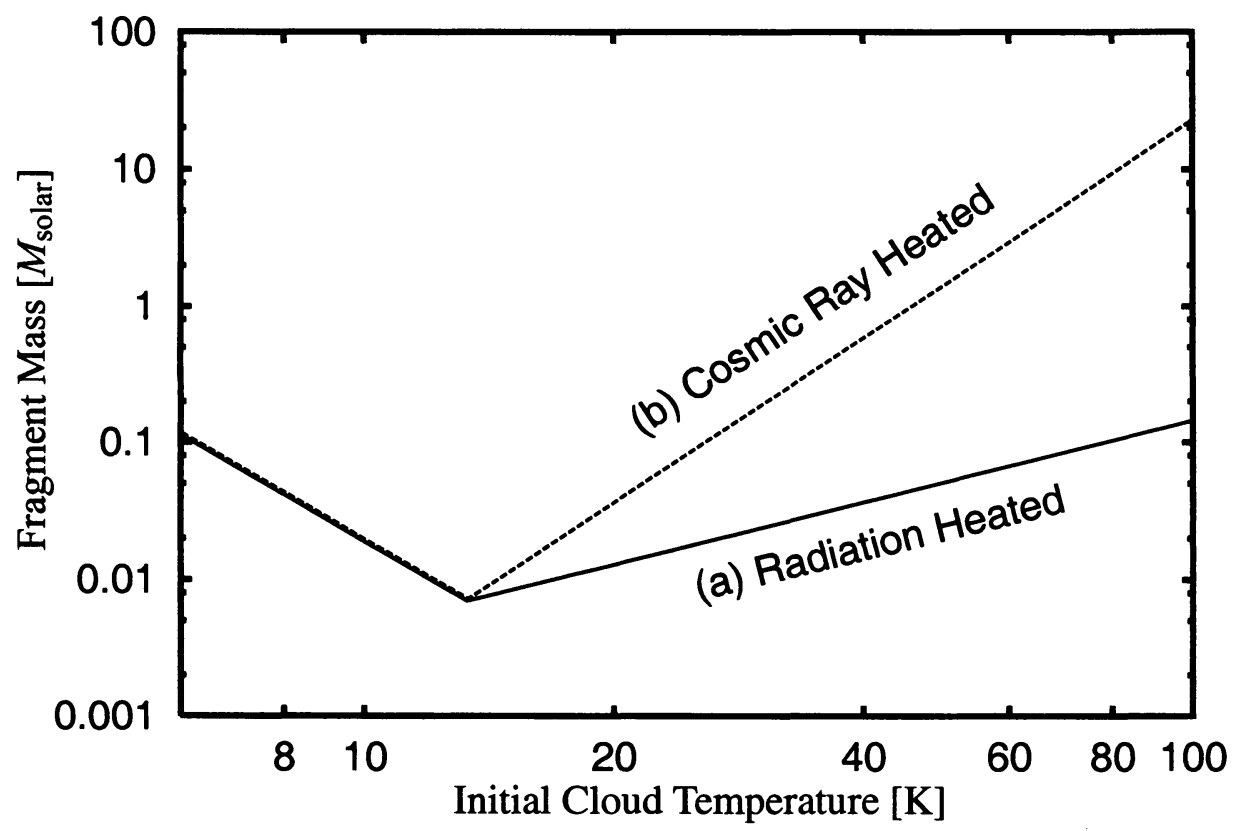

Figure 1. Minimum fragment mass versus initial cloud temperature: (a) stellar radiation heated cases (solid line) and (b) cosmic ray heated cases (dotted line). The minimum fragment mass depends on the initial cloud temperature and the nature of heating sources. The universal minimum mass, which is about $0.007 M_{\odot}$, is given when the initial cloud temperature is 13.3 $\mathrm{K}$.

Inutsuka, S., \& Miyama, S. M. 1997, ApJ, 480, 681 (IM97)

Luhman, K. L., Rieke, G. H., Young, T., Cotera, A. S., Chen, H., Rieke, M. J., Schneider, G., \& Thompson R. I. 2000, ApJ, 540, 1016

Masunaga, H., \& Inutsuka, S. 1999, ApJ, 510, 822

Miyama, S. M., Narita, S., \& Hayashi, C. 1984, Prog. Theor. Phys., 78, 1273

Ogochi, K., \& Nakamoto, T. 2000, ApJ, submitted

Rees, M. 1976, MNRAS, 176, 483

Silk, J. 1977, ApJ, 214, 152

Zapatero Osorio, M. R., Béjar, V. J. S., Rebolo, R., Martin, E. L., \& Basri, G. 1999, ApJ, 524, L115 